\title{
The Hippocampus Modulates Dopamine Neuron Responsivity by Regulating the Intensity of Phasic Neuron Activation
}

\author{
Daniel J Lodge ${ }^{*, I}$ and Anthony A Grace' \\ 'Departments of Neuroscience, Psychiatry, and Psychology, University of Pittsburgh, Pittsburgh, PA, USA
}

\begin{abstract}
Aberrant dopamine (DA) signaling has been advanced as a contributing factor to the pathophysiology of a number of psychiatric conditions including schizophrenia; however, the many factors involved in regulating DA system responsivity have not been completely delineated to date. We have shown previously that DA neuron activity states are independently regulated by distinct afferent pathways. We now provide evidence that these pathways interact to control the population of neurons that are phasically activated. As shown previously, infusions of NMDA into the ventral subiculum (vSub) increases the number of spontaneously active DA neurons (population activity), while having no effect on firing rate or average bursting activity. In contrast, NMDA activation of the pedunculopontine tegmental nucleus (PPTg) results in a significant increase in DA neuron burst firing without significantly affecting population activity. However, simultaneous excitation of the vSub and PPTg induces a significant increase in both DA neuron population activity and burst firing resulting in a $\sim 4$-fold increase in the number of high-bursting neurons observed per electrode track. These data suggest that DA neuron population activity is not simply associated with the tonic release of DA in forebrain regions, but rather represents a recruitable pool of DA neurons that can be further modulated by excitatory inputs to induce a graded phasic response. Taken as a whole, we propose that the synchronous activity of distinct afferent inputs to the VTA phasically activates selective populations of DA neurons, and hence may be a site of pathological regulation underlying aberrant DA signaling.

Neuropsychopharmacology (2006) 3 I, | 356-136I. doi:I 0. I038/sj.npp. I 300963; published online 23 November 2005
\end{abstract}

Keywords: dopamine; electrophysiology; ventral subiculum; hippocampus; pedunculopontine tegmental nucleus; schizophrenia

\section{INTRODUCTION}

The tonic/phasic model of dopamine (DA) release was first proposed as a model for defining the operating modes of the DA system and regulation of its responsivity (Grace, 1991). This model proposed that mesolimbic DA release is regulated via two independent mechanisms: (1) transient or 'phasic' DA release that is mediated mainly through burst firing of the neuron and (2) extra synaptic or 'tonic' levels of DA which are mediated by basal DA neuron activity and regulated via presynaptic inputs (Floresco et al, 2003; Grace, 1991). Thus, DA neuron burst firing induces a large transient increase in synaptic DA (Chergui et al, 1994) and is considered to be the functionally relevant signal sent to postsynaptic sites to encode reward prediction or indicate incentive salience (Berridge and Robinson, 1998; Schultz, 1998). In contrast, tonic DA transmission occurs on

*Correspondence: Dr DJ Lodge, Department of Neuroscience, University of Pittsburgh, 446 Crawford Hall, Pittsburgh, PA I5260, USA, Tel: + I 412624 0824, Fax: + 14126249198 ,

E-mail: Lodge@bns.pitt.edu

Received 17 August 2005; revised 3 October 2005; accepted 4 October 2005

Online publication: 12 October 2005 at http://www.acnp.org/citations/ Npp I 0 | 2050505 | 9/default.pdf a much slower scale and has been suggested to set the background level of DA receptor activation; as such, it has been proposed to regulate the responsivity of the DA system through pre- and postsynaptic mechanisms (Grace, 1991).

Since this time, there has been increasing evidence for functionally related but independent afferent systems regulating either tonic or phasic DA release (Floresco et al, 2003). Thus, we have shown that a decrease in tonic GABAergic transmission to the VTA results in a significant and selective increase in DA neuron population activity that corresponds to increases in tonic DA efflux in the nucleus accumbens (Acb) (Floresco et al, 2003). In contrast, activation of excitatory afferents to the VTA results in a selective increase in DA neuron burst firing that is associated with a significant increase in phasic DA transmission (Floresco et al, 2003). Moreover, each of these transmission states can differentially affect afferent input to the Acb (Goto and Grace, 2005).

One important feature of this regulation is that activation of excitatory inputs to the VTA results in burst firing only in DA neurons that are already spontaneously active (Floresco et al, 2003). Thus, in those neurons that are inactive, presumably due to GABA-mediated hyperpolarization, activation of glutamatergic afferents has little or no effect, likely attributable to $\mathrm{Mg}^{2+}$ blockade of the NMDA 
receptor-channel complex. These findings suggest the presence of a unique interdependence of the GABAergic and glutamatergic VTA inputs, that is, only neurons that are not under GABA-mediated hyperpolarization are capable of entering a burst firing mode in response to a glutamatergic input. We now propose that changes in DA neuron population activity may not simply regulate extrasynaptic DA levels in forebrain regions but may also reflect which DA neurons are capable of exhibiting phasic activation in response to glutamatergic input.

The ventral subiculum (vSub) is the major output relay of the hippocampus, a structure vital for the encoding and recall of episodic memory (Squire, 1992). Moreover, it has been demonstrated that stimulation of the vSub results in a significant $(\sim 2$-fold) increase in DA neuron population activity, which is correlated with an increase in extracellular DA levels in the Acb (Blaha et al, 1997; Brudzynski and Gibson, 1997; Floresco et al, 2001, 2003; Legault and Wise, 1999). This ability of subiculum stimulation to selectively increase the number of spontaneously active DA neurons has been shown to be due to a multisynaptic pathway (Floresco et al, 2001). Thus, vSub activation excites Acb projection neurons that inhibit ventral pallidal GABAergic afferents to the VTA, thereby releasing DA neurons from a tonic inhibitory influence (Floresco et al, 2001). In contrast, the pedunculopontine tegmental nucleus (PPTg) is a glutamatergic/cholinergic region driven by a number of limbic afferents, including the prefrontal cortex, bed nucleus of the stria terminalis and central nucleus of the amygdala (Semba and Fibiger, 1992), and has been shown to be activated by auditory (Pan and Hyland, 2005; Reese et al, 1995), visual (Pan and Hyland, 2005) and somatosensory stimuli (Grunwerg et al, 1992). The PPTg was found to also directly regulate burst firing of DAergic neurons (Floresco et al, 2001; Lokwan et al, 1999). These previous studies focused on the independent actions of afferent systems regulating tonic and phasic DA neuron activity. In this study, we explore the possibility that these systems may actually act in concert with respect to regulating DA neuron activity states, with the vSub determining which subsets of DA neurons may be phasically activated via PPTg inputs.

\section{METHODS}

All experiments were performed in accordance with the guidelines outlined in the National Institute of Health Guide for the Care and Use of Laboratory Animals and were approved by the Institutional Animal Care and Use Committee of the University of Pittsburgh.

\section{Surgery and Pharmacological Manipulations}

Male Sprague-Dawley rats (31 rats in total: 250-400 g) were anesthetized with chloral hydrate $(400 \mathrm{mg} / \mathrm{kg}$, intraperitoneally) and placed in a stereotaxic apparatus. Chloral hydrate was utilized since, under this anesthetic, DA neuron activity states more closely resemble that observed in freely moving rats (Hyland et al, 2002). Anesthesia was maintained by supplemental administration of chloral hydrate as required to maintain suppression of limb compression withdrawal reflex and a core body temperature of $37^{\circ} \mathrm{C}$ was sustained by a thermostatically controlled heating pad. For acute administration of NMDA/vehicle, rats were implanted with $23 \mathrm{G}$ injection cannulae $2.0 \mathrm{~mm}$ dorsal to the vSub (A/P $-6.0, \mathrm{M} / \mathrm{L}+5.3, \mathrm{D} / \mathrm{V}-4.5 \mathrm{~mm}$ from bregma) and/or PPTg $(\mathrm{A} / \mathrm{P}-8.0, \mathrm{M} / \mathrm{L}+1.6, \mathrm{D} / \mathrm{V}-5.0 \mathrm{~mm}$ from bregma) that were fixed in place with dental cement and two anchor screws. $N$-methyl-D-aspartate (Sigma, St Louis, MO) was dissolved in Dulbecco's phosphate-buffered saline (PBS) and infused $(0.75 \mu \mathrm{g} / 0.5 \mu \mathrm{l}$; duration $\sim 30 \mathrm{~s})$ through a $30-\mathrm{G}$ injection cannula protruding $2.0 \mathrm{~mm}$ past the end of the guide. The efficacy of NMDA infusions was demonstrated by a potent increase in firing rate of vSub neurons after intra-vSub NMDA compared to Dulbecco's PBS infusions (vehicle $1.0 \pm 0.4 \mathrm{~Hz}$ : NMDA $5.3 \pm 0.9 \mathrm{~Hz}, p<0.05$, MannWhitney rank-sum test, $n=5$ rats). The control group for DA neuron population studies consisted of seven rats that received vehicle (Dulbecco's PBS) infusions into either the vSub, PPTg, or both regions. These groups all showed similar DA neuron population activity parameters so that their data were combined. All rats received only one injection per region and DA cell recordings were typically recorded from $10 \mathrm{~min}$ to $2 \mathrm{~h}$ following infusions.

\section{Extracellular Recordings}

Glass extracellular microelectrodes (impedance 6-14 M $\Omega$ ) were lowered into the VTA $(\mathrm{A} / \mathrm{P}-5.3, \mathrm{M} / \mathrm{L}+0.8 \mathrm{~mm}$ from bregma and -6.5 to $-9.0 \mathrm{~mm}$ ventral of brain surface) using a hydraulic microdrive, and the activity of the population of DA neurons was determined by counting the number of spontaneously active DA neurons encountered while making five to nine vertical passes, separated by $200 \mu \mathrm{m}$, throughout the VTA. Spontaneously active DA neurons were identified using previously established electrophysiological criteria (Grace and Bunney, 1983), and once isolated, their activity was recorded for 2-3 min. Three parameters of activity were measured: (i) population activity (defined as the number of spontaneously active DA neurons recorded per electrode track), (ii) basal firing rate, and (iii) the proportion of action potentials occurring in bursts (defined as the occurrence of two spikes with an interspike interval of $<80 \mathrm{~ms}$, and the termination of the burst defined as the occurrence of an interspike interval of $>160 \mathrm{~ms}$; Grace and Bunney, 1983).

\section{Histology}

At the cessation of the experiment, the recording site was marked via iontophoretic ejection of Pontamine sky blue from the tip of the recording electrode $(30 \mu \mathrm{A}$ constant current: $20-30 \mathrm{~min}$ ). After dye injection, rats were killed by an overdose of anesthetic, decapitated and their brains removed, fixed for at least $48 \mathrm{~h}(8 \%(\mathrm{w} / \mathrm{v})$ paraformaldehyde in PBS), and cryoprotected (25\% (w/v) sucrose in PBS) until saturated. Brains were sectioned ( $60 \mu \mathrm{m}$ coronal sections), mounted onto gelatin-chrom alum-coated slides and stained with cresyl violet for histochemical verification of electrode sites and thionin for verification of cannula sites. All histology was performed with reference to a stereotaxic atlas (Paxinos and Watson, 1986) and (for PPTg placements) alternating brain slices stained for NADPH-diaphorase (a marker for cholinergic neurons; Vincent et al, 1983) 


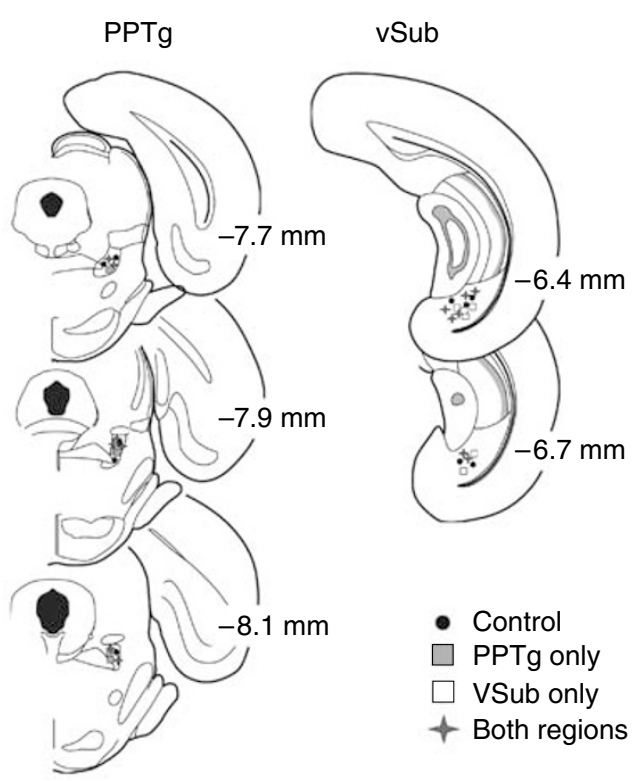

Figure I Histological localization of injection sites in the vSub and/or PPTg. Numbers beside each plate represent approximate A/P distance from bregma.

and thionin. The locations of injection sites are presented in Figure 1 .

\section{Analysis}

Electrophysiological analysis of DA neuron activity was performed using custom-designed computer software (Neuroscope). All data are represented as the mean \pm standard error of the mean (SEM). Differences in standard electrophysiological recording parameters were examined using a one-way analysis of variance (ANOVA) followed by a Dunnett's post hoc test, whereas differences in the proportion of high- and low-bursting neurons were examined using a two-way ANOVA followed by a Student-Newman-Keuls post hoc test. The criteria for defining high and low burst firing neurons was determined from a bimodal Gaussian fit of the burst firing frequency distribution curve obtained with simultaneous PPTg and vSub activation. The cutoff values are defined as $2 \mathrm{SD}$ from the means of each peak. As such, low-bursting neurons are defined as having $<33 \%$ action potentials fired in burst (mean $13.6 \pm 9.9 \%$ ), while high-bursting defined by $>56 \%$ (mean $69.1 \pm 6.8 \%$ ) spikes fired in burst.

All statistics were calculated using the SigmaStat software program (Jandel), while the bimodal Gaussian fit was determined using Origin Pro (OriginLab).

\section{RESULTS}

Rats that received control vehicle infusions ( $n=7$ rats, 65 neurons) exhibited an average of $1.18 \pm 0.1$ spontaneously active DA neurons per electrode track that fired at an average rate of $3.9 \pm 0.2 \mathrm{~Hz}$ with $29 \pm 3 \%$ of action potentials fired in bursts (Figure 2a-c), consistent with previous findings (Floresco et al, 2001, 2003). Intrasubiculum infusion of NMDA ( $n=6$ rats, 86 neurons) resulted in a
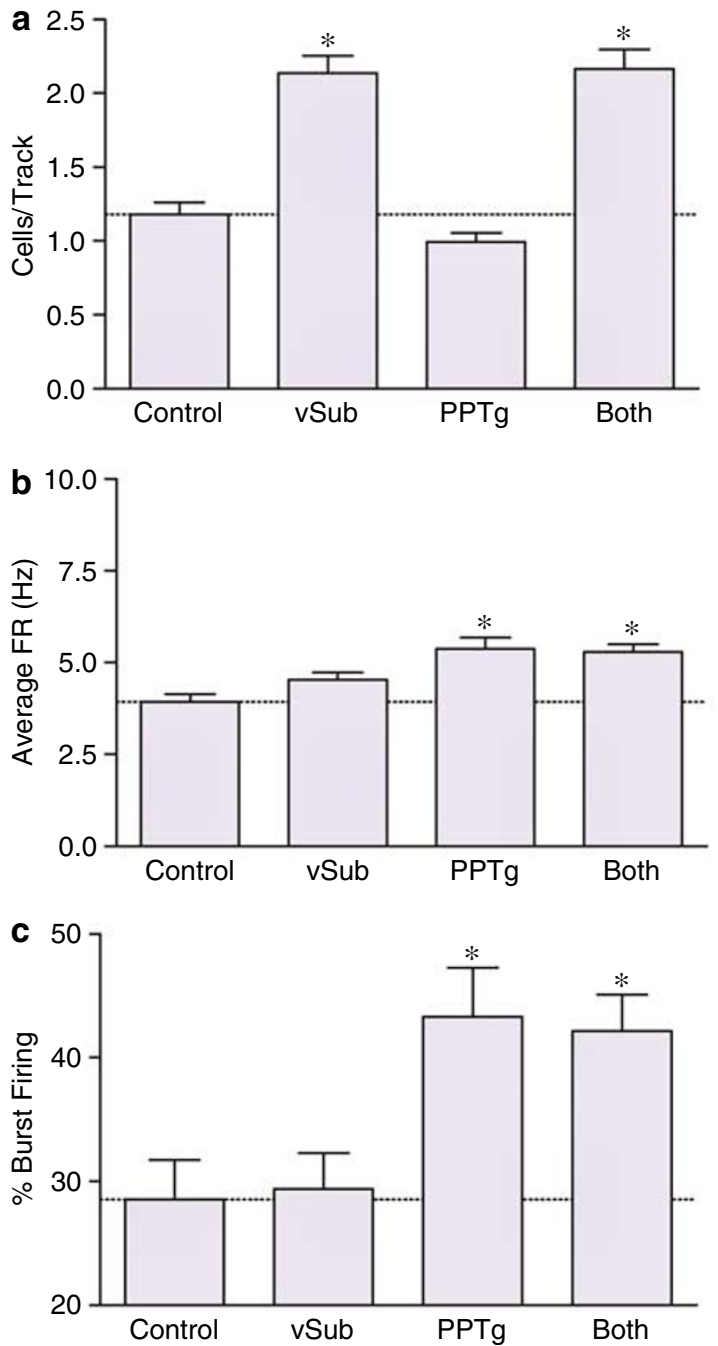

Figure 2 NMDA infusions alter population activity and firing pattern of VTA DA neurons. NMDA $(0.75 \mu \mathrm{g} / 0.5 \mu \mathrm{l})$ was infused into the vSub, PPTg, or both regions, and the activity of spontaneously active VTA dopamine neurons examined. Three parameters of activity were recorded: population activity (a), average firing rate (b), and average percent burst firing (c). *Statistically significant difference from control (vehicle infusions) $(p<0.05$ one-way ANOVA; Dunnett's post hoc: $n=6-7$ rats/group).

significant $(\sim 2$-fold, $P<0.05)$ increase in $D A$ neuron population activity (defined as number of neurons active; Figure 2a) without significantly affecting average burst firing or firing rate relative to control (Figure $2 b$ and $c$ ). Analysis of the burst firing frequency distribution curves demonstrated no significant differences between control and vSub activation groups, further demonstrating that $\mathrm{vSub}$ activation does not modulate average DA neuron burst firing. Furthermore, vSub stimulation induced a concomitant ( 2-fold) increase in both high- and low-bursting neurons, operationally defined on the basis of distribution of burst events across the population of neurons recorded, as neurons with $>56$ or $<33 \%$ action potentials in bursts, respectively (see Methods; Figure 3).

Activation of a glutamatergic/cholinergic pathway to the VTA by an intra-PPTg infusion of NMDA ( $n=6$ rats, 45 neurons) resulted in significantly more burst firing of these cells (Figure 2c) without significantly affecting population 


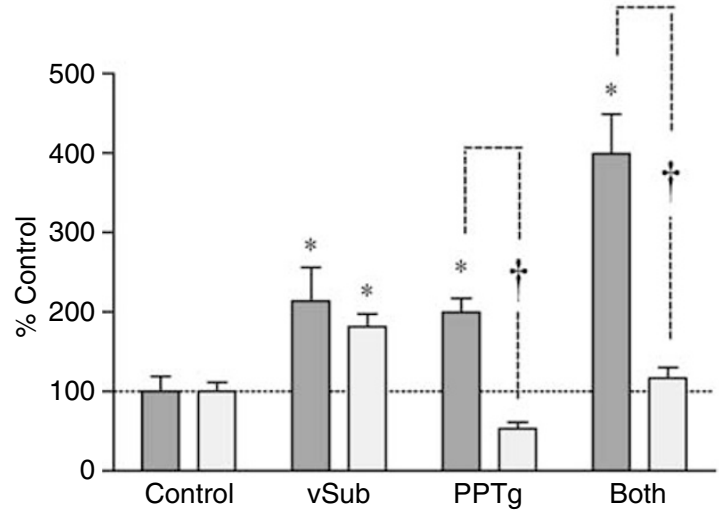

Figure 3 Activation of the PPTg and the vSub by NMDA has additive effects on the number of high-bursting DA neurons recorded in the VTA. NMDA $(0.75 \mu \mathrm{g} / 0.5 \mu \mathrm{l})$ was infused into the vSub, PPTg, or both regions, and the activity of spontaneously active VTA dopamine neurons examined. Dark bars represent high-bursting neurons defined as cells firing $>56 \%$ action potentials in burst, while light bars represent low-bursting neurons $(<33 \%$ action potentials in burst). *Significant difference compared to control (vehicle infusions); ${ }^{\dagger}$ significant difference between high- and lowbursting neurons $(p<0.05$ two-way ANOVA; Student-Newman-Keuls post hoc: $n=6-7$ rats/group).

activity (Figure 2a). This transition from low to high burst firing is reflected in a $\sim 2$-fold increase in high-bursting neurons (Figure 3).

Simultaneous activation of the PPTg and vSub ( $n=7$ rats, 101 neurons) resulted in a significant increase in both DA neuron population activity and average burst firing (Figure $2 \mathrm{a}$ and c). Interestingly, the net effect of this activation was a four-fold increase in the number of high-bursting neurons per electrode track (Figure 3).

It is important to note that both PPTg and simultaneous vSub/PPTg infusions of NMDA resulted in a significant increase in average firing rate (Figure $2 \mathrm{~b}$ ) and is likely reflective of the increased burst firing induced by these treatments (Grace and Bunney, 1984).

\section{DISCUSSION}

Using in vivo extracellular recordings in chloral hydrate anesthetized rats, we report that although individual manipulations of afferent pathways exert independent control over DA neuron firing characteristics, simultaneous activation of multiple regions can result in a supraphasic increase in DAergic transmission. Specifically, the present study confirms that infusions of NMDA into the vSub induce an increase in the number of spontaneously active DA neurons (population activity), while having no effect on firing rate or average bursting activity. In contrast, NMDA activation of the PPTg resulted in a significant increase in DA neuron burst firing without affecting population activity. Interestingly, the simultaneous excitation of the vSub and PPTg induced a significant increase in both DA neuron population activity and average burst firing resulting in a $\sim 4$-fold increase in the number of high-bursting neurons observed per electrode track. These data suggest that DA neuron population activity is not only associated with the tonic release of DA in forebrain regions but also

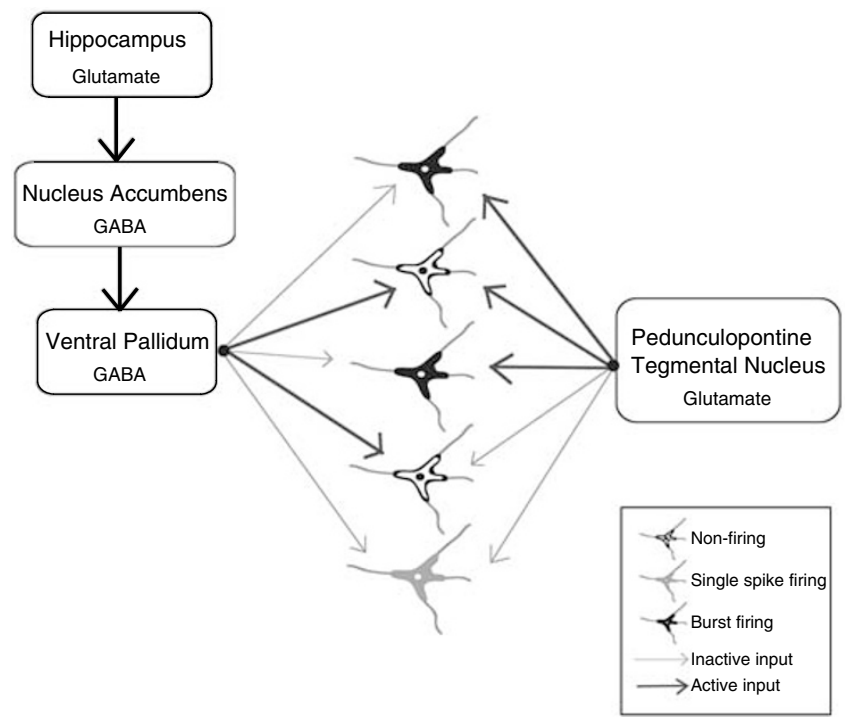

Figure 4 A model to explain how selective neural circuits enable the hippocampus to selectively modulate the population of DA neurons capable of exhibiting phasic activation. Stimulation of hippocampal afferents excites Acb projection neurons that inhibit VP GABAergic afferents to the VTA, thereby releasing DA neurons from a tonic inhibitory influence. Concurrent PPTg activation directly influences burst firing only in DA neurons that have been removed from the tonic GABA-mediated hyperpolarization.

represents a recruitable pool of DA neurons that can be further modulated by excitatory inputs to induce a graded phasic response. Taken as a whole, we hypothesize that the synchronous activity of select afferent inputs to the VTA are required to induce the most robust DA signal and hence pathologies of afferent systems may result in aberrant DA signaling. Moreover, the hippocampus subiculum would be positioned to select which subpopulations of DA neurons are capable of exhibiting phasic activation by the PPTg, thereby providing independent means for regulating DA neuron output (Figure 4).

As previously demonstrated, vSub activation by NMDA resulted in a $\sim 2$-fold increase in the number of spontaneously active DA neurons observed per electrode track without affecting percent bursting or average firing rate. The finding that a concomitant increase in both the number of high- and low-bursting neurons was observed suggests that quiescent DA neurons are subject to the same tonic regulation as spontaneously active neurons once they are released from GABA-mediated inhibition. Interestingly, PPTg activation induced a similar $\sim 2$-fold increase in the number of high-bursting DA neurons albeit through a different mechanism, that is, a transition from low to high burst firing resultant from the increased glutamatergic (and possibly cholinergic) tone to the VTA as suggested by previous studies of glutamate afferents and DA neuron burst firing (Lokwan et al, 1999; Smith and Grace, 1992). The present study demonstrates that simultaneous activation of the vSub and PPTg results in a summation of these effects resulting in a $\sim 4$-fold increase in the number of high-bursting neurons, confirming the interdependence of the GABAergic and glutamatergic inputs to the ventral mesencephalon. 
Schultz and others have shown that presentation of reward-related signals induces transient burst firing in DA neurons only when the reward-related signal is unpredicted (ie novel) (Schultz, 1998). Furthermore, the PPTg has been shown to respond to sensory stimuli (Grunwerg et al, 1992; Reese et al, 1995) and it has been suggested that this region may be involved in signal detection independent of its behavioral salience (Pan and Hyland, 2005); therefore, the PPTg may be involved in detecting a signal with the hippocampus evaluating its novelty (Lisman and Grace, 2005). Since the computation of novelty requires the comparison of incoming information with stored memories, this likely occurs in the hippocampus (Knight, 1996). As such, it is possible that novelty detection by the hippocampus gates information processing in the VTA by amplifying the DA neuron response to excitatory inputs. This ability of the hippocampus to gate DAergic responses may have important implications for understanding the etiology of schizophrenia, which has been proposed to be associated with both hippocampal dysfunction and DA dysregulation (Grace, 2000; Harrison, 2004). Evidence for a DA involvement in schizophrenia was initially based on the efficacy of DA antagonists in treating the disease and more recently on the increased amphetamine-induced DA release observed in schizophrenia patients (Laruelle and AbiDargham, 1999). The hippocampus has been implicated in this disease due to a reduction in volume, enhancement of basal metabolism, and deficits in hippocampal memory processes observed in schizophrenia patients (Harrison, 2004). One possible consequence of this pathologically enhanced hippocampal activity (given the results of the current study) may be an increased salience of previously insignificant stimuli, that is, it is plausible that hippocampal damage such as that observed in schizophrenia may result in an increased DA neuron population activity that may lead to a considerably amplified phasic DA signal in response to nonsalient events or objects (Kapur, 2003).

\section{Conclusions}

Taken as a whole, the present study demonstrates that although individual manipulations of afferent pathways exert independent control over DA neuron firing characteristics, the synchronous activity of multiple regions is required to induce the most robust DA signal. Moreover, that modulation of DA neuron population activity is not only associated with altered tonic DA levels in forebrain regions but also appears to determine which subsets of the DA neuron population may be further modulated by excitatory inputs to induce a graded phasic response. These data further suggest that pathologies of afferent systems, such as the hippocampus, may result in aberrant DA signaling and could explain why schizophrenia patients fail to differentiate relatively nonsalient stimuli.

\section{ACKNOWLEDGEMENTS}

We would like to thank Niki MacMurdo and Christy Smolak for their valuable technical assistance, and Brian Lowry for the production, development, and assistance with the custom-designed electrophysiology software (Neuroscope).
This work was supported by the USPHS DA15408 and MH57440.

\section{REFERENCES}

Berridge KC, Robinson TE (1998). What is the role of dopamine in reward: hedonic impact, reward learning, or incentive salience? Brain Res Rev 28: 309-369.

Blaha CD, Yang CR, Floresco SB, Barr AM, Phillips AG (1997). Stimulation of the ventral subiculum of the hippocampus evokes glutamate receptor-mediated changes in dopamine efflux in the rat nucleus accumbens. Eur J Neurosci 9: 902-911.

Brudzynski SM, Gibson CJ (1997). Release of dopamine in the nucleus accumbens caused by stimulation of the subiculum in freely moving rats. Brain Res Bull 42: 303-308.

Chergui K, Suaud-Chagny MF, Gonon F (1994). Nonlinear relationship between impulse flow, dopamine release and dopamine elimination in the rat brain in vivo. Neuroscience 62: 641-645.

Floresco SB, Todd CL, Grace AA (2001). Glutamatergic afferents from the hippocampus to the nucleus accumbens regulate activity of ventral tegmental area dopamine neurons. J Neurosci 21: 4915-4922.

Floresco SB, West AR, Ash B, Moore H, Grace AA (2003). Afferent modulation of dopamine neuron firing differentially regulates tonic and phasic dopamine transmission. Nat Neurosci 6: $968-973$.

Goto Y, Grace AA (2005). Dopaminergic modulation of limbic and cortical drive of nucleus accumbens in goal-directed behavior. Nat Neurosci 8: 805-812.

Grace AA (1991). Phasic versus tonic dopamine release and the modulation of dopamine system responsivity: a hypothesis for the etiology of schizophrenia. Neuroscience 41: 1-24.

Grace AA (2000). Gating of information flow within the limbic system and the pathophysiology of schizophrenia. Brain Res Rev 31: $330-341$.

Grace AA, Bunney BS (1983). Intracellular and extracellular electrophysiology of nigral dopaminergic neurons - 1. Identification and characterization. Neuroscience 10: 301-315.

Grace AA, Bunney BS (1984). The control of firing pattern in nigral dopamine neurons: burst firing. J Neurosci 4: 2877-2890.

Grunwerg BS, Krein H, Krauthamer GM (1992). Somatosensory input and thalamic projection of pedunculopontine tegmental neurons. Neuroreport 3: 673-675.

Harrison PJ (2004). The hippocampus in schizophrenia: a review of the neuropathological evidence and its pathophysiological implications. Psychopharmacologia 174: 151-162.

Hyland BI, Reynolds JN, Hay J, Perk CG, Miller R (2002). Firing modes of midbrain dopamine cells in the freely moving rat. Neuroscience 114: 475-492.

Kapur S (2003). Psychosis as a state of aberrant salience: a framework linking biology, phenomenology, and pharmacology in schizophrenia. Am J Psychiatry 160: 13-23.

Knight R (1996). Contribution of human hippocampal region to novelty detection. Nature 383: 256-259.

Laruelle M, Abi-Dargham A (1999). Dopamine as the wind of the psychotic fire: new evidence from brain imaging studies. J Psychopharmacol 13: 358-371.

Legault M, Wise RA (1999). Injections of $N$-methyl-D-aspartate into the ventral hippocampus increase extracellular dopamine in the ventral tegmental area and nucleus accumbens. Synapse 31: 241-249.

Lisman J, Grace AA (2005). The hippocampal-VTA loop: controlling the entry of information into long-term memory. Neuron 46: 703-713.

Lokwan SJA, Overton PG, Berry MS, Clark D (1999). Stimulation of the pedunculopontine tegmental nucleus in the rat produces 
burst firing in A9 dopaminergic neurons. Neuroscience 92: 245-254.

Pan W-X, Hyland BI (2005). Pedunculopontine tegmental nucleus controls conditioned responses of midbrain dopamine neurons in behaving rats. $J$ Neurosci 25: 4725-4732.

Paxinos G, Watson C (1986). The Rat Brain in Stereotaxic Coordinates. Academic Press: Australia, Sydney.

Reese NB, Garcia-Rill E, Skinner RD (1995). Auditory input to the pedunculopontine nucleus: II. Unit responses. Brain Res Bull 37: 265-273.

Schultz W (1998). Predictive reward signal of dopamine neurons. J Neurophysiol 80: 1-27.
Semba K, Fibiger HC (1992). Afferent connections of the laterodorsal and the pedunculopontine tegmental nuclei in the rat: a retro- and antero-grade transport and immunohistochemical study. J Comp Neurol 323: 387-410.

Smith ID, Grace AA (1992). Role of the subthalamic nucleus in the regulation of nigral dopamine neuron activity. Synapse 12: 287-303.

Squire LR (1992). Memory and the hippocampus: a synthesis from findings with rats, monkeys, and humans. Psychological Rev 99: 195-231.

Vincent SR, Satoh K, Armstrong DM, Fibiger HC (1983). NADPHdiaphorase: a selective histochemical marker for the cholinergic neurons of the pontine reticular formation. Neurosci Lett 43: 31-36. 